\title{
Use of Fecal Calprotectin as A Predictor of Spontaneous Bacterial Peritonitis in Post Hepatitis C Cirrhotic Egyptian Patients
}

Atef Abou Elfotouh Ibrahim, Mohammed Salah Ali Hussein, Zakarya Mohamed Zakarya Shady, Mahmoud Mohammed Mohammed Metwally, Abdelrahman Saad Mohamed Amer

Department of Internal Medicine, Faculty of Medicine, Al-Azhar University, Cairo, Egypt

*Corresponding author: Abdelrahman Saad Amer; Mobile: (+20)01008677734; Email: abdosaad123@gmail.com

\begin{abstract}
Background: patients with liver cirrhosis have an increased risk of infections mainly spontaneous bacterial peritonitis, which is present in about $15 \%$ of patients with cirrhosis and ascites. This is believed to be mainly due to bacterial translocation (BT) although accurate mechanisms of BT are unknown, SIBO, gut dysmotility, increased intestinal permeability and impaired defense mechanisms are regarded as major risk factors for BT.

Objective: to assess the value of fecal calprotectin (FC) as a predictor of SBP in cirrhotic Egyptian patients.

Patients and Methods: this study included 75 subjects; they were divided into two groups: 25 cirrhotic subjects with ascites and no SBP as control group and 50 with liver cirrhosis and ascites and an evident SBP, all patients were subjected to full history taking, complete clinical examination and routine laboratory investigations and microbiological analysis of ascitic fluid, stool samples were collected for measuring FC by ELISA.

Results: FC showed a highly significant difference $(\mathrm{p}<0.001)$ with the median FC in SBP case group higher than median FC of cirrhotic control group and both groups above average normal range, also a colleration emerged between elevated FC and previous history of hepatic encephalopathy in case group.

Conclusion: FC was significantly elevated in cirrhotic patients dependant on the severity of liver disease as assessed by Child pugh score and a significant colleration emerged between elevated FC and complications of liver cirrhosis as SBP; So FCC can be used as a useful marker for early prediction and diagnosis of SBP in patient with liver cirrhosis.
\end{abstract}

Keywords: Fecal calprotectin, Spontaneous bacterial peritonitis, Hepatitis C cirrhotic.

\section{INTRODUCTION}

Patients with liver cirrhosis have an increased risk of infections mainly spontaneous bacterial peritonitis (SBP) which is present in approximately $15 \%$ of patients with cirrhosis and ascites. This is believed to be mainly due to bacterial translocation $(\mathrm{BT})^{(\mathbf{1})}$.

BT refers to the entry of viable bacteria or their products into the regional lymph nodes, the systemic circulation, and possibly extra intestinal organs. The origin of such microorganisms is the enteric flora and translocation occurs via a defective mucosal barrier ${ }^{(2)}$.

Over $70 \%$ of these cases are caused by intestinal bacteria and can be prevented by selective bacterial decontamination with non absorbed or absorbed antibiotics, which confirms that, the gut is the main source of microorganisms. Although accurate mechanisms of BT are unknown, small intestinal bacterial overgrowth (SIBO), gut dysmotility, increased intestinal permeability and impaired defense mechanisms are regarded as major risk factors for BT (3).

Calprotectin is calcium and zinc binding protein that belongs to the S-100 protein group and it has been estimated that it may account for more than $60 \%$ of the soluble cytosolic proteins in human neutrophil granulocytes, it increases under conditions such as inflammation, infection and malignancy. Calprotectin can be measured in stool, plasma, synovial fluid, cerebrospinal fluid, oral fluids and urine ${ }^{(4)}$.
Calprotectin measurements in fecal samples not only correlate with the degree of neutrophil migration in the gastrointestinal mucosa, but also serve as reliable surrogate marker of intestinal inflammation $^{(5)}$.

Gundling study is considered one of the most important studies regarding the role of fecal calprotectin in the diagnosis of BT, They confirmed that patients with cirrhosis had significantly elevated fecal calprotectin levels when compared to healthy controls. Moreover, this increase correlated with the severity of liver. Even more significantly, higher calprotectin values were associated with advanced stages of HE, the presence of SBP, as well as extraintestinalinfections ${ }^{(6)}$.

In all, calprotectin remains a promising surrogate marker for BT in cirrhosis. It demonstrates many advantages, especially in its fecal measurement, as it is a non-invasive, quick and relatively easy to perform assay, with proven clinical value in other disease states ${ }^{(7)}$.

\section{AIM OF THE WORK}

It is to assess the value of fecal calprotectin (FC) as a predictor of spontaneous bacterial peritonitis in cirrhotic Egyptian patients.

\section{PATIENTS AND METHODS}

The study data: A prospective, case control study, which have been conducted in association with the G.I. Unit of Elhussein University Hospital in Cairo, Egypt. 
The study was approved by the Ethics Board of Al- 3- Laboratory investigations.

Azhar University and an informed written consent 4- Measurement of fecal calprotectin concentration: was taken from each participant in the study.

\section{Patients}

$>$ This study included the following groups:

- Twenty five patients with liver cirrhosis, ascites and with no clinical or investigational evidence of SBP as a control group.

- Fifty patients with liver cirrhosis, ascites and an evidenced spontaneous bacterial peritonitis.

\section{Exclusion Criteria:}

$>$ All patients with diseases that could have caused an abnormal FC including:

- Active GIT bleeding.

- All patients who report having diarrhea as GIT infections may cause elevated fecal calprotectin.

- Coeliac disease.

- Colo-rectal carcinoma.

- Certain drugs (e.g. non-steroidal anti-inflammatory drugs, anticoagulants, antibiotic therapy, proton pump inhibitors).

- Food allergies as: cow's milk, eggs, fish, wheat and others.

- Inflammatory bowel diseases (IBDs): Crohn's disease and ulcerative colitis.

- Ongoing alcohol abuse.

\section{Methods:}

All patients were subjected to:

1- Full history.

2- Complete clinical examination.
Fecal Calprotectin was assayed by a commercial enzyme linked immuno-sorbent assay (ELISA) method according the manufacturer's instructions (human CALPRO ELISA kit, Sunnyvale, CA, USA).

Each patient (including both groups of case SBP patients and cirrhotic controls) provided a single stool sample collected within $48 \mathrm{~h}$ of hospital admission; samples obtained for FC measurement were collected in caped disposable plastic containers and stored at -20 degree $\mathrm{C}$ until they were assayed.

Test principle: CALPRO using polyclonal antibody against calprotectin in an enzyme linked immuno-sorbent assay system.

Calprotectin present in the diluted sample is bound by the antibody adsorbed to the surface of the plastic well.

The enzyme conjugated antibody binds to the captured antigen and subsequently the enzyme catalyses the conversion of the substrate to a colored product. The intensity of the colour is proportional to the amount calprotectin concentration captured in the diluted samples

5- Abdominal ultrasound (US).

6- Colonoscopy was done in highly susceptible patients.

\section{RESULTS}

Table (1): Comparison between the two studied groups according to demographic data

\begin{tabular}{|c|c|c|c|c|c|c|}
\hline & \multicolumn{2}{|c|}{$\begin{array}{l}\text { Control } \\
(n=25)\end{array}$} & \multicolumn{2}{|c|}{$\begin{array}{c}\text { Cases } \\
(\mathbf{n}=\mathbf{5 0})\end{array}$} & \multirow[t]{2}{*}{$\chi^{2}$} & \multirow[t]{2}{*}{$\mathbf{p}$} \\
\hline & No. & $\%$ & No. & $\%$ & & \\
\hline $\begin{array}{l}\text { Gender: } \\
\text { Male } \\
\text { Female } \\
\end{array}$ & $\begin{array}{l}15 \\
10 \\
\end{array}$ & $\begin{array}{l}60.0 \\
40.0 \\
\end{array}$ & $\begin{array}{l}33 \\
17 \\
\end{array}$ & $\begin{array}{l}66.0 \\
34.0 \\
\end{array}$ & 0.260 & 0.610 \\
\hline $\begin{array}{l}\text { Age (years): } \\
\text { Min. - Max. } \\
\text { Mean } \pm \text { SD. } \\
\text { Median }\end{array}$ & \multicolumn{2}{|c|}{$\begin{array}{c}43.0-70.0 \\
57.64 \pm 7.86 \\
57.0\end{array}$} & \multicolumn{2}{|c|}{$\begin{array}{c}37.0-80.0 \\
60.66 \pm 9.53 \\
62.0\end{array}$} & $\begin{array}{c}\mathrm{t}=1.36 \\
8\end{array}$ & 0.176 \\
\hline
\end{tabular}

$\chi^{2}$ : Chi square test t: Student t-test, $\quad$ p: $\mathrm{p}$ value for comparing between the studied groups

Seventy five patients with liver cirrhosis were included in the study the control group consisting of 25 patients, with liver cirrhosis, ascites and with no evident SBP, 15 of them were males and 10 were females. Their mean age was $57.64 \pm 7.86$, while the case group consisting of 50 patients, with liver cirrhosis, ascites and an evident SBP, 33 of them was males and 17 were females. Their mean age was $60.66 \pm 9.53$. The cause of liver disease in all included patients of both case and control groups were HCV infection (table 1). 
Table (2): Comparison between the two studied groups according to Fecal Calprotectin (mg/kg)

\begin{tabular}{|l|c|c|c|c|}
\hline Fecal Calprotectin (mg/kg) & $\begin{array}{c}\text { Control } \\
(\mathbf{n = 2 5})\end{array}$ & $\begin{array}{c}\text { Cases } \\
(\mathbf{n = 5 0})\end{array}$ & $\mathbf{U}$ & $\mathbf{p}$ \\
\hline Min. - Max. & $32.0-66.0$ & $65.0-472.0$ & & \\
Mean \pm SD. & $57.63 \pm 8.12$ & $196.51 \pm 118.65$ & $3.0^{*}$ & $<0.001^{*}$ \\
Median & 59.50 & 131.0 & & \\
\hline
\end{tabular}

$\mathbf{U}$ : Mann Whitney test p: p value for comparing between the studied groups

*: Statistically significant at $\mathrm{p} \leq 0.05$

Comparison between the studied groups as regard to Fecal Calprotectin $(\mathrm{mg} / \mathrm{kg})$ it showed a statistically highly significance in between as in the control group it ranged from 32 to $66(\mathrm{mg} / \mathrm{kg})$ with median 59.5 , and in the case group it ranged from 65 to $472(\mathrm{mg} / \mathrm{kg})$ with median $131(\mathrm{p}<0.001)$ (table 2).

Table (3): Relation between Child pugh score and Fecal Calprotectin ( $\mathrm{mg} / \mathrm{kg})$ in case group and total sample

\begin{tabular}{|l|c|c|c|c|}
\hline \multirow{2}{*}{ Fecal Calprotectin (mg/kg) } & \multicolumn{2}{|c|}{ Child pugh score } & \multirow{2}{*}{ U } & \multirow{2}{*}{ p } \\
\cline { 2 - 5 } & $\mathbf{B}$ & $\mathbf{C}$ & & \\
Case group: & $(\mathbf{n}=\mathbf{1 9})$ & $(\mathbf{n = 3 1 )}$ & & \\
Min. - Max. & $65.0-425.0$ & $78.0-472.0$ & & \\
Mean \pm SD. & $129.84 \pm 102.98$ & $238.73 \pm 109.38$ & $98.50^{*}$ & $<0.001^{*}$ \\
Median & 88.0 & 259.0 & & \\
\hline Total sample: & $(\mathbf{n}=\mathbf{3 1})$ & $(\mathbf{n = 4 4 )}$ & & \\
Min. - Max. & $32.0-425.0$ & $42.0-472.0$ & & \\
Mean \pm SD. & $101.94 \pm 87.60$ & $186.95 \pm 123.85$ & $420.0^{*}$ & $0.010^{*}$ \\
Median & 73.0 & 130.50 & & \\
\hline
\end{tabular}

U: Mann Whitney test, p: p value for association between Child pugh score and Fecal Calprotectin

*: Statistically significant at $\mathrm{p} \leq 0.05$

Relation between Child pugh score and Fecal Calprotectin showed a positive correlation and a statistically highly significance as in the case group the median value of Fecal Calprotectin measurement was $88 \mathrm{mg} / \mathrm{kg}$ in Child B patients and $259 \mathrm{mg} / \mathrm{kg}$ in Child $\mathrm{C}$ patients $(\mathrm{P}<0.001)$ (table 3 ).

Table (4): ROC curve for Calprotectin ( $\mathrm{mg} / \mathrm{kg})$ to predict cases group

\begin{tabular}{|c|c|c|c|c|c|c|c|c|}
\hline & AUC & P & $\mathbf{9 5 \%}$ C.I & Cut off & Sensitivity & Specificity & PPV & NPV \\
\hline $\begin{array}{c}\text { Calprotectin } \\
\text { (mg/kg) }\end{array}$ & 0.97 & $<0.001^{*}$ & $0.992-1.003$ & $>\mathbf{6 5}$ & $\mathbf{9 5 . 9 2}$ & 95.83 & 97.9 & 92.0 \\
\hline
\end{tabular}

AUC: Area Under a Curve

NPV: Negative predictive value p value: Probability value

PPV: Positive predictive value
CI: Confidence Intervals

*: Statistically significant at $\mathbf{p} \leq \mathbf{0 . 0 5}$

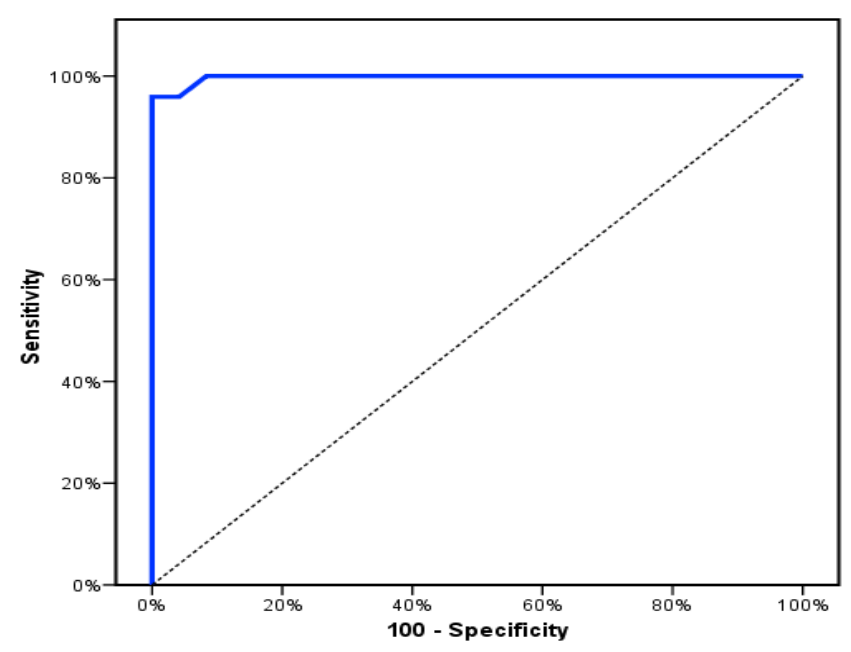

Figure (1): ROC curve for Calprotectin $(\mathrm{mg} / \mathrm{kg})$ to predict cases group

When we use FC in prediction of SBP, FC had sensitivity of $95 \%$ and specificity of $95 \%$ with PPV and NPV of $97 \%$ and $92 \%$ respectively with a cut off value of $>65 \mathrm{mg} / \mathrm{kg}$ with AUC of 0.97 in prediction of SBP group (table 7). 
Table (5): Comparison between the two studied groups according to Total bilirubin

\begin{tabular}{|l|c|c|c|c|}
\hline Total bilirubin (mg/dl) & $\begin{array}{c}\text { Control } \\
(\mathbf{n = 2 5})\end{array}$ & $\begin{array}{c}\text { Cases } \\
(\mathbf{n = 5 0})\end{array}$ & $\begin{array}{c}\text { Test of } \\
\text { Sig. }\end{array}$ & p \\
\hline Min. - Max. & $0.80-5.20$ & $0.80-10.50$ & \multirow{2}{*}{$\mathrm{U}=442}$. & \multirow{2}{*}{$0.039^{*}$} \\
\hline Mean \pm SD. & $1.69 \pm 0.83$ & $2.47 \pm 2.01$ & 0 & \\
\hline Median & 1.60 & 1.80 & $0^{*}$ & \\
\hline
\end{tabular}

t: Student t-test U: Mann Whitney test p: p value for comparing between the studied groups

Comparison between the studied groups as regard to Total bilirubin it showed a statistically highly significance in between as in the control group it ranged from .8 to $5.2(\mathrm{mg} / \mathrm{dl})$ with median 1.6 , and in the case group it ranged from .8 to $10.5(\mathrm{mg} / \mathrm{dl})$ with median 1.8 (table $\mathbf{8})$.

Table (6): Relation betweenhistory of hepatic encephalopathy and FecalCalprotectin ( $\mathrm{mg} / \mathrm{kg})$ in cases group

\begin{tabular}{|l|c|c|c|c|}
\hline \multirow{2}{*}{ Fecal Calprotectin (mg/kg) } & \multicolumn{2}{|c|}{$\begin{array}{c}\text { History of hepatic } \\
\text { encephalopathy }\end{array}$} & \multirow{2}{*}{$\mathbf{U}$} & $\mathbf{p}$ \\
\cline { 2 - 3 } & $\mathbf{N o}$ & $\mathbf{P r e v}$ & & \\
\hline Cases group: & $\mathbf{( n = 3 9 )}$ & $(\mathbf{n}=\mathbf{1 1})$ & & \\
Min. - Max. & $65.0-472.0$ & $253.0-371.0$ & & \\
Mean \pm SD. & $164.24 \pm 114.47$ & $308.0 \pm 38.22$ & $62.50^{*}$ & $<0.001^{*}$ \\
Median & 114.50 & 305.0 & & \\
Total sample: & $\mathbf{( n = 6 4 )}$ & $(\mathbf{n}=\mathbf{1 1})$ & & \\
Min. - Max. & $32.0-472.0$ & $253.0-371.0$ & & \\
Mean \pm SD. & $122.97 \pm 103.51$ & $308.0 \pm 38.22$ & $62.50^{*}$ & $<0.001^{*}$ \\
Median & 81.0 & 305.0 & & \\
\hline
\end{tabular}

U: Mann Whitney test p: p value for association between history Of Hepatic Encephalopathy and Fecal Calprotectin

*: Statistically significant at $\mathrm{p} \leq 0.05$

Relation between the history of hepatic encephalopathy and Fecal Calprotectin showed a positive correlation and a statistically highly significance as in the case group the median value of Calprotectin measurement was 114.5 $\mathrm{mg} / \mathrm{kg}$ in patients who didn't experience a previous history of hepatic Encephalopathy, and a median value of $305 \mathrm{mg} / \mathrm{kg}$ in patients who had a previous history of hepatic Encephalopathy before $(\mathrm{P}<0.001)$ (table 6).

\section{DISCUSSION}

The median FCCs were significantly elevated in SBP case group $(131 \mathrm{mg} / \mathrm{kg})$ while in cirrhotic control group it was $(59.5 \mathrm{mg} / \mathrm{kg})$ this median FCCs is relatively high and above the normal range according to the manufacturer's instructions, despite careful exclusion of other causes of abnormal calprotectin results, e.g. GI bleeding, diarrhea, alcohol intake and drugs intake e.g. antibiotics, NSIAD and anticoagulants.

When we use FC in prediction of SBP, FC had sensitivity of $95 \%$ and specificity of $95 \%$ with PPV and NPV of $97 \%$ and $92 \%$ respectively with a cut off value of $>65 \mathrm{mg} / \mathrm{kg}$ with AUC of 0.97 in prediction of SBP group

In our study, we found no significant influence (p 0.301) of laboratory parameters of systemic inflammation (WBC count) on FCCs in our case (SBP) group in comparison with our control group (cirrhotics). Therefore the elevation of FCCs may be caused by a primary intestinal inflammation which is not secondarily the result of a systemic inflammatory reaction. Likewise Gundling et $\boldsymbol{a l} .{ }^{(\boldsymbol{)})}$ who reported that fecal calprotectin levels were elevated in cirrhotic patients with SBP $(275.4 \mathrm{mg} / \mathrm{kg})$ and in cirrhotic patients with no SBP $(65.8 \mathrm{mg} / \mathrm{kg})$ compared to noncirrhotic controls $(17.5 \mathrm{mg} / \mathrm{kg})$.

Gundling et $\boldsymbol{a l}^{(\boldsymbol{( 6 )}}$ also found no significant influence $(\mathrm{P}=0.142$ and $\mathrm{P}=0.207)$ of laboratory parameters of systemic inflammation (CRP, WBC count) on FCCs in cirrhotic subgroup. Therefore, elevated FCCs in cirrhotic patients as opposed to controls may be caused by a regional (primary) intestinal inflammation which is not secondarily the result of a systemic inflammatory reaction.

In our study, patients were divided according to the severity of liver disease as assessed by Child Pugh score into three groups, child A, B and C. the elevated FCCs in this study showed a significantly positive correlation with the severity of liver disease ( $p<0.001)$. In the case group there was no patients in child A group and the mean FCCs in child B $(129.84 \pm 102.98 \mathrm{mg} / \mathrm{kg})$ with a median $(88 \mathrm{mg} / \mathrm{kg})$ and in child $\mathrm{C}$ was $(238.73 \pm 109.38 \mathrm{mg} / \mathrm{kg})$ with a median $(259 \mathrm{mg} / \mathrm{kg})$ respectively compared to a mean of $(57.63 \pm 8.12 \mathrm{mg} / \mathrm{kg})$ with a median $(59.5 \mathrm{mg} / \mathrm{kg})$ in control group.

Fecal calprotectin in cirrhosis was firstly investigated by Yagmur et al.$^{(8)}$ who found a significant elevation in FCCs in patients with advanced chronic liver disease (Child-C) and additionally added that there 
was a trend towards higher levels of fecal calprotectin in subjects with alcoholic cirrhosis.

However, Montalto et al. ${ }^{\left({ }^{(9)}\right.}$ who performed a longitudinal study of active-drinking alcoholics found no significant differences in median FCCs between alcoholics and controls. Interestingly Yagmur et al. ${ }^{(\mathbf{8})}$ described in their study the highest of all concentrations in two cirrhotic patients with SBP. In accordance with Yagmur $\boldsymbol{e t} \boldsymbol{a l}{ }^{(\mathbf{8})}$ in the present study FCCs were significantly elevated dependent on the severity of liver disease as assessed by Child-Pugh score.

Homann et al. ${ }^{(10)}$ investigated the prognostic value of plasma and ascites calprotectin in cirrhosis. They demonstrated that plasma calprotectin was a highly significant marker of poor survival in alcohol induced cirrhosis as high calprotectin concentration was significantly associated with poor survival.

The same authors found no association between increased plasma calprotectin concentrations and the severity of liver disease. Furthermore, homann firstly described that high plasma calprotectin levels may characterize a group of cirrhotics with recurring bacterial infections ${ }^{(\mathbf{1 0})}$.

In our study, when comparing the relation between FCCs and the previous history of hepatic encephalopathy in SBP case group subjects we found that median FCCs were significantly higher in subjects with previous hepatic encephalopathy $\mathrm{n}=11$ (305 $\mathrm{mg} / \mathrm{kg}$ ) than subjects without previous history of hepatic encephalopathy $\mathrm{n}=39(114.5 \mathrm{mg} / \mathrm{kg})(\mathrm{p}<0.001)$.

Likewise Gundling et al. ${ }^{(\boldsymbol{( 6 )}}$ found a significant association emerged between elevated FCCs and hepatic encephalopathy grading as measured by Westhaven criteria Differentiating grading of (0 and 1$)$ from (2 and 3) resulted in a sensitivity of $94 \%$ and a specificity of $95 \%(\mathrm{P}<0.001)$ using an optimal cut point of $164 \mathrm{mg} / \mathrm{kg}$. And concluded that FC may serve as a screening tool to identify cirrhotic patients with hepatic encephalopathy.

Therefore, as Gundling $\boldsymbol{e t} \boldsymbol{a l} .{ }^{(\boldsymbol{(})}$ concluded, FCCs may serve as a screening tool to identify cirrhotic patients with SBP. However, further studies are needed to investigate FCCs prospectively in cirrhotic patients with ascites and SBP before and after medical treatment in comparison to standard diagnostic procedures.

\section{CONCLUSION}

This study has demonstrated that FC was significantly elevated in cirrhotic patients dependant on the severity of liver disease as assessed by Child pugh score and a significant colleration emerged between elevated FC and complications of liver cirrhosis as SBP;
So fecal calprotectin concentration (FCCs) can be used as a useful marker for early prediction and diagnosis of SBP in patient with liver cirrhosis.

\section{RECOMMENDATION}

We recommend that FCCs can be used as a valid parameter as it is a simple, non-invasive and rapid screening test to make a diagnosis and assessment of the severity of SBP.

Further studies are needed to investigate FCCs in cirrhotic patients with SBP before and after medical treatment to find out whether FCCs might be a useful parameter for monitoring the further course. Since the prognostic significance of calprotectin in plasma and ascites fluid was already confirmed.

The studies in the future should include determination of plasma and ascites calprotectin as a reference method

\section{REFERENCES}

1. Almeida J, Galhenage S, Yu J et al. (2012): Gut flora and BT in chronic liver disease. World J Gastroenterol., 12(10):1493-502.

2. Berg RD, Garlington AW, Dal Pont E et al. (2011): Translocation of certain indigenous bacteria from the gastrointestinal tract to the mesenteric lymph nodes and other organs. J Hepatol., 103: 2007-14.

3. Rasaratnam B, Connelly N and Chin-Dusting J (2014): Nitricoxide and the hyperdynamic circulation in cirrhosis; is there a role for selective intestinal decontamination? Clin Sci., 107: 425-434.

4. Beşer OF, Sancak S, Erkan T et al. (2014): Can Fecal Calprotectin Level Be Used as a Markers of Inflammation in the Diagnosis and Follow-Up of Cow's Milk Protein Allergy? Allergy Asthma Immunol Res., 6(1):33-38.

5. Burri E, Beglinger C (2014): The use of fecal calprotectin as a biomarker in gastrointestinal disease. Expert Rev Gastroenterol Hepatol., 8: 197-210

6. Gundling F, Schmidtler F, Hapfelmeier A et al. (2011): Fecal calprotectin is a useful screening parameter for hepatic encephalopathy and spontaneous bacterial peritonitis in cirrhosis. Liver Int., 31(9):1406-15.

7. Montalto M, Gallo A, Santoro L et al. (2013): Role of fecal calprotectin in gastrointestinal disorders. Eur Rev Med Pharmacol Sci., 17: 1569-1582.

8. Yagmur E, Schnyder B, ScholtenD et al. (2006): Elevated concentrations of fecal calprotectin in patients with liver cirrhosis. Dtsch. Med. Wochenschr., 131:19301934.

9. Montalto M, Gallo A, FerrulliA et al. (2011): Fecal calprotectin concentrations in alcoholic patients: a longitudinal study. Eur J Gastroenterol Hepatol., 23(1):76-80.

10. Homann C, Christensen E, Schlichting P et al. (2003): Ascites fluid and plasma calprotectin concentrations in liver disease. Scand J Gastroenterol., 38(4):415-20. 